\title{
Long-term monitoring of selected radio sources ${ }^{\star}$
}

\author{
B. Peng ${ }^{1,2}$, A. Kraus ${ }^{1}$, T.P. Krichbaum ${ }^{1}$, and A. Witzel ${ }^{1}$ \\ 1 Max-Planck-Institut für Radioastronomie, Auf dem Hügel 69, 53121 Bonn, Germany \\ 2 Beijing Astronomical Observatory, National Astronomical Observatories, Chinese Academy of Sciences, Beijing 100012, China
}

Received January 27; accepted March 21, 2000

\begin{abstract}
The Effelsberg $100 \mathrm{~m}$ radio telescope has been used, over a period of 5.5 years, to monitor the flux densities of 40 extragalactic radio sources, including the complete sample of 13 flat spectrum sources from the S5 Survey, and various other active sources. The study of their long-term variability characteristics at several wavelengths, the source light curves, spectra, and a statistical analysis are presented and discussed in this paper.
\end{abstract}

Key words: galaxies: active - BL Lacertae objects: general - quasars: general — radio continuum: galaxies

\section{Introduction}

Since the detections of high-frequency variability (Kellermann \& Pauliny-Toth 1968) and low-frequency variability (e.g. Hunstead 1972), many extragalactic sources have been found to show significant variability at various frequencies, on time scales of months to a few years (e.g. Dennison et al. 1981), and even of a day (Witzel et al. 1986; Heeschen et al. 1987). Both relativistic bulk motion (Rees 1966a; Jones \& Burbidge 1973; Burbidge et al. 1974; Blandford \& Königl 1979) and refractive interstellar scintillation (e.g. Rickett 1986) are commonly invoked to explain the various variability phenomena detected.

In order to study the long-term variability behavior of extragalactic radio sources over a range of wavelengths, several monitoring programs have been carried out (Spangler \& Cotton 1981; Aller et al. 1985; Padrielli et al. 1987; Salonen et al. 1987; Bregman et al. 1990; Teräsranta et al. 1992; Bloom et al. 1999). We have carried out flux monitoring of selected sources over a wide range of wavelengths with the Effelsberg $100 \mathrm{~m}$ radio telescope since 1994 . Here we present the results of this long-term

Send offprint requests to: B. Peng, e-mail: pb@class1.bao.ac.cn

* Figures 1 to 30 are only available in electronic version at http://www.edpsciences.org monitoring for 40 sources at several wavelengths. Among them, 13 compact extragalactic radio sources, with a declination $\delta \geq 70^{\circ}$, a galactic latitude $\left|b^{\mathrm{II}}\right| \geq 10^{\circ}$, flux density at $5 \mathrm{GHz} S_{5 \mathrm{GHz}} \geq 1 \mathrm{Jy}$, and spectral index (between $\lambda=11 \mathrm{~cm}$ and $\lambda=6 \mathrm{~cm}) \alpha_{6}^{11} \leq 0.5\left(S \sim \nu^{-\alpha}\right)$, form a complete sample of flat spectrum sources from the S5 Survey (Kühr et al. 1981).

In the following section, we briefly introduce the observations and data reduction. In Sect. 3 the results for all 40 extragalactic radio sources are presented, including light curves, spectra and statistics. Subsequently, we make brief comments on individual sources. Observational findings are summarized in Sect. 5. Throughout the paper the radio spectral index is defined by $S_{\nu} \propto \nu^{-\alpha}$, a deceleration parameter $q_{0}=0.5$ and a Hubble constant of $H_{0}=50 \mathrm{~km} \mathrm{~s}^{-1} \mathrm{Mpc}^{-1}$ are used.

\section{Observations and data reduction}

We present observations performed from January 1994 to July 1999 with the Effelsberg $100 \mathrm{~m}$ radio telescope, at wavelengths $\lambda=21,18,11,6,3.6,2.8,2,1.3 \mathrm{~cm}, 9$ and $7 \mathrm{~mm}$. One should note, that both time and frequency coverage are rather irregular, depending on the availability of time slots and receivers. Most observations have been made at wavelengths of $11,6,2.8$, and $1.3 \mathrm{~cm}$.

All sources monitored are listed in Table 1 with their optical identifications, galactic latitudes, and redshifts (taken from Kühr et al. 1981 and Stickel et al. 1994). Further, we give the estimated spectral indices $\alpha_{6}^{11}$ and $\alpha_{2.8}^{6}$, and - if available from the literature - the superluminal velocity for the fastest component (see individual references). We also note whether a source showed Intraday variability (IDV, e.g. Wagner \& Witzel 1995; Kraus 1997) at least once or not.

Since all target sources are point-like and moderately strong $(\gtrsim 0.2 \mathrm{Jy})$ at the frequencies of observation, we were able to perform the measurements with cross-scans (in Azimuth and Elevation). As a first step of the analysis, 
Table 1. Source properties: Opt. Id. denotes the optical identification, $b^{\mathrm{II}}$ the galactic latitude, $z$ the redshift, $\alpha\left(\alpha_{6}^{11}, \alpha_{2.8}^{6}\right)$ spectral indices, $\beta_{\mathrm{a}}$ apparent superluminal speed (in unit of speed of light $c$ ), Ref. reference for $\beta_{\mathrm{a}}$, and IDV or not

\begin{tabular}{|c|c|c|c|c|c|c|c|c|c|}
\hline Source & Other name & Opt. Id. & $b^{11}$ & $z$ & $\alpha_{6}^{11}$ & $\alpha_{2.8}^{6}$ & $\beta_{\mathrm{a}}$ & Ref. & IDV \\
\hline $0016+731$ & $*$ & QSO & $+10.7^{\circ}$ & 1.781 & $+0.06 \pm 0.94$ & $-0.07 \pm 0.62$ & 16.6 & S92 & + \\
\hline $0134+329$ & $3 \mathrm{C} 48$ & QSO & $-28.7^{\circ}$ & 0.367 & $+0.89 \pm 0.02$ & $+1.02 \pm 0.02$ & & & \\
\hline $0153+744$ & $*$ & QSO & $+12.4^{\circ}$ & 2.338 & $+0.72 \pm 0.06$ & $+0.97 \pm 0.05$ & 29.4 & W88 & + \\
\hline $0212+735$ & $*$ & QSO & $+12.0^{\circ}$ & 2.367 & $-0.26 \pm 0.18$ & $-0.04 \pm 0.14$ & 36.8 & W88 & - \\
\hline $0235+164$ & & BLL & $-39.1^{\circ}$ & 0.940 & $+0.16 \pm 1.39$ & $-0.57 \pm 1.31$ & 54.0 & Chu & + \\
\hline $0316+413$ & $3 \mathrm{C} 84$ & GAL & $-13.3^{\circ}$ & 0.017 & $+0.28 \pm 0.25$ & $+0.19 \pm 0.18$ & 0.4 & $\mathrm{R} 84$ & - \\
\hline $0420-014$ & & QSO & $-33.1^{\circ}$ & 0.915 & $-0.26 \pm 0.19$ & $-0.24 \pm 0.19$ & & & $?$ \\
\hline $0454+844$ & $*$ & BLL & $+24.7^{\circ}$ & 0.112 & $-0.05 \pm 0.42$ & $+0.18 \pm 0.35$ & $>3.2$ & S92 & $?$ \\
\hline $0518+165$ & $3 \mathrm{C} 138$ & QSO & $-11.3^{\circ}$ & 0.759 & $+0.68 \pm 0.02$ & $+0.78 \pm 0.02$ & & & \\
\hline $0528+134$ & & QSO & $-11.0^{\circ}$ & 2.060 & $-0.74 \pm 0.53$ & $-0.16 \pm 0.67$ & 12.4 & B99 & $?$ \\
\hline $0538+498$ & 3C 147 & QSO & $+10.3^{\circ}$ & 0.545 & $+0.88 \pm 0.02$ & $+0.91 \pm 0.03$ & & & \\
\hline $0615+820$ & $*$ & QSO & $+26.0^{\circ}$ & 0.710 & $+0.21 \pm 0.09$ & $+0.37 \pm 0.10$ & $<4.4 ?$ & S92 & - \\
\hline $0716+714$ & $*$ & BLL & $+28.0^{\circ}$ & $0.30 ?$ & $-0.05 \pm 0.76$ & $-0.13 \pm 0.86$ & 4.6 & $\mathrm{~S} 92$ & + \\
\hline $0735+178$ & & BLL & $+18.1^{\circ}$ & 0.424 & $+0.30 \pm 0.29$ & $+0.58 \pm 0.39$ & $>14.4$ & M90 & + \\
\hline $0804+499$ & & QSO & $+32.6^{\circ}$ & 1.433 & $+0.03 \pm 0.50$ & $-0.13 \pm 0.70$ & & & + \\
\hline $0835+580$ & & QSO & $+36.9^{\circ}$ & 1.534 & $+1.09 \pm 0.03$ & $+1.20 \pm 0.06$ & -0.7 & H93 & $?$ \\
\hline $0836+710$ & $*$ & QSO & $+34.4^{\circ}$ & 2.172 & $+0.34 \pm 0.20$ & $+0.14 \pm 0.38$ & 21.6 & O98 & + \\
\hline $0851+202$ & OJ 287 & BLL & $+35.8^{\circ}$ & 0.306 & $-0.27 \pm 0.33$ & $-0.15 \pm 0.32$ & 6.4 & M90 & + \\
\hline $0917+624$ & & QSO & $+41.0^{\circ}$ & 1.446 & $-0.15 \pm 0.17$ & $+0.02 \pm 0.16$ & 7.8 & S96 & + \\
\hline $0923+392$ & $4 \mathrm{C} 39.25$ & QSO & $+46.2^{\circ}$ & 0.698 & $-1.01 \pm 0.08$ & $-0.23 \pm 0.06$ & 7.1 & S87 & - \\
\hline $0951+699$ & & GAL & $+40.6^{\circ}$ & 0.001 & $+0.69 \pm 0.02$ & $+0.76 \pm 0.02$ & & & - \\
\hline $0954+658$ & & BLL & $+43.1^{\circ}$ & 0.367 & $-0.07 \pm 0.58$ & $-0.05 \pm 0.49$ & 12.4 & G96 & + \\
\hline $1039+811$ & $*$ & QSO & $+34.7^{\circ}$ & 1.254 & $-0.04 \pm 0.19$ & $-0.41 \pm 0.14$ & 35.6 & W88 & $?$ \\
\hline $1150+812$ & $*$ & QSO & $+35.8^{\circ}$ & 1.250 & $+0.07 \pm 0.21$ & $-0.01 \pm 0.10$ & 7.0 & W88 & + \\
\hline $1226+023$ & $3 \mathrm{C} 273$ & QSO & $+64.4^{\circ}$ & 0.158 & $+0.23 \pm 0.12$ & $+0.19 \pm 0.17$ & 16.1 & $\mathrm{C} 87$ & - \\
\hline $1253-055$ & 3C 279 & QSO & $+57.1^{\circ}$ & 0.536 & & $-0.62 \pm 0.43$ & 18.4 & C79 & - \\
\hline $1328+307$ & $3 \mathrm{C} 286$ & QSO & $+80.7^{\circ}$ & 0.849 & $+0.59 \pm 0.02$ & $+0.68 \pm 0.02$ & & & \\
\hline $1409+524$ & $3 \mathrm{C} 295$ & GAL & $+60.8^{\circ}$ & 0.461 & $+1.05 \pm 0.02$ & $+1.21 \pm 0.02$ & & & \\
\hline $1458+718$ & 3C 309.1 & QSO & $+42.1^{\circ}$ & 0.905 & $+0.57 \pm 0.09$ & $+0.48 \pm 0.11$ & 13.1 & K90 & - \\
\hline $1641+399$ & $3 \mathrm{C} 345$ & QSO & $+40.9^{\circ}$ & 0.594 & $-0.03 \pm 0.07$ & $+0.08 \pm 0.11$ & 18.9 & $\mathrm{BM}$ & - \\
\hline $1652+398$ & & BLL & $+38.9^{\circ}$ & 0.034 & $+0.13 \pm 0.07$ & $+0.17 \pm 0.05$ & & & + \\
\hline $1739+522$ & & QSO & $+31.7^{\circ}$ & 1.379 & $-0.13 \pm 0.83$ & $-0.04 \pm 0.69$ & & & + \\
\hline $1749+701$ & $*$ & BLL & $+30.7^{\circ}$ & 0.770 & $+0.05 \pm 0.17$ & $+0.06 \pm 0.23$ & 4.7 & M90 & + \\
\hline $1803+784$ & $*$ & BL? & $+29.1^{\circ}$ & 0.684 & $-0.18 \pm 0.24$ & $-0.05 \pm 0.15$ & 7.8 & $\mathrm{~S} 92$ & + \\
\hline $1823+568$ & & BLL & $+26.1^{\circ}$ & 0.664 & $-0.23 \pm 0.21$ & $-0.02 \pm 0.31$ & 5.1 & G89 & + \\
\hline $1928+738$ & $*$ & QSO & $+23.5^{\circ}$ & 0.302 & $-0.01 \pm 0.09$ & $+0.06 \pm 0.10$ & 14.1 & E85 & + \\
\hline $2007+777$ & $*$ & BLL & $+22.7^{\circ}$ & 0.342 & $-0.04 \pm 0.32$ & $+0.05 \pm 0.27$ & 4.7 & M90 & + \\
\hline $2105+420$ & NGC 7027 & $\mathrm{PN}$ & $-3.5^{\circ}$ & & $-0.67 \pm 0.02$ & $-0.12 \pm 0.02$ & & & \\
\hline $2200+420$ & BLLAC & BLL & $-10.4^{\circ}$ & 0.069 & $-0.08 \pm 0.47$ & $-0.04 \pm 0.36$ & 7.5 & M90 & + \\
\hline $2251+158$ & 3C 454.3 & QSO & $-38.2^{\circ}$ & 0.859 & $-0.14 \pm 0.19$ & $+0.29 \pm 0.27$ & 17.7 & $\mathrm{PT}$ & - \\
\hline
\end{tabular}

Notes for columns in Table 1 - for Other name, a $\star$ marks the source which belongs to the S5 survey; for Opt. Id., PN stands for Planetary Nebulae; for $z$ and $\alpha_{6}^{11}$, blank if it is not available; for IDV, + if it shows at least once IDV, - if not, ? if unclear yet, blank if it is a primary flux calibrator source.

Notes for references in Table 1 - BM: Biretta et al. 1986, B99: Britzen et al. 1999, C87: Cohen et al. 1987, C79: Cotton et al. 1979, Chu: Chu et al. 1996, E85: Eckart et al. 1985, G89: Gabuzda et al. 1989, G96: Gabuzda et al. 1996, H93: Hough et al. 1993, K90: Kus et al. 1990, M90: Mutel 1990, O98: Otterbein et al. 1998, PT: Pauliny-Toth et al. 1987, R84: Romney et al. 1984, S87: Shaffer et al., 1987, S92: Schalinski et al. 1992, S96: Standke et al. 1996, W88: Witzel et al. 1988.

a Gaussian was fitted to every cross-scan. The average amplitude of these Gaussians (which are the result of the convolution of the point-like source with the antenna-beam) is a measure of the source flux density. Subsequently, we corrected for the elevation-dependence of the gain of the telescope and systematic time-dependent effects. To determine those, we frequently observed steep-spectrum sources (and the primary calibrators) which were known to show no variability on short timescales (cf. Quirrenbach et al. 1992; Kraus 1997; Kraus et al., in preparation).

Finally, we linked our observations to an absolute flux density scale (Baars et al. 1977) by observing primary calibrators such as 3C 286 and 3C 48. The measurement errors are composed of the statistical errors from averaging the 
individual samples in a scan, and a contribution from the calibration errors, which are reflected by apparent residual fluctuations of the non-variable sources.

\section{Results}

In the monitoring period of 5.5 years, the flux densities of the primary calibrators, 3C 48, 3C 138, 3C 147, 3C 286 , 3C 295, and NGC 7027 (and also few other sources, as reported in Sect. 3.2), remained constant as expected at a level of about $2 \%$ at wavelength $\lambda \geq 2.8 \mathrm{~cm}$, and about $3 \%$ at $\lambda=1.3 \mathrm{~cm}$.

\subsection{Light curves and source spectra}

Light curves for each source are plotted in Figs. 1-20 at the four best observed wavelengths $11,6,2.8$ and $1.3 \mathrm{~cm}$. In case there are less than 3 data points at a given wavelength, the plot has been omitted. We make brief comments on individual sources in Sect. 4.

Spectra of all sources are presented in Figs. 21-25, by taking all fluxes measured at each wavelength over the 5.5 year monitoring period. Obviously, the flux variations of a source are reflected in its spectrum. Although the reliability of the spectra suffer from flux density variations, it is interesting to note that,

- the spectra of the flat spectrum sources $0153+744$ and 1253-055 steepen for $\lambda \leq 11 \mathrm{~cm}$, and $0735+178$ probably steepens for $\lambda \leq 6 \mathrm{~cm}$;

- the spectra of sources $0316+413$ and $0615+820$ are inverted at $\lambda \leq 11 \mathrm{~cm}, 0212+735,0454+844,1641+399$, $1928+738,2007+777$ and $2251+158$ inverted at $\lambda \leq$ $6 \mathrm{~cm}, 0917+624$ at $\lambda \leq 3.6 \mathrm{~cm}, 1803+784$ at $\lambda \leq 2 \mathrm{~cm}$;

- sources 0420-014, 0528+134, 0836+710, 0851+202, $1226+023$ and $2105+420$ (NGC 7027) have flat spectra at $\lambda \leq 6 \mathrm{~cm}$, and $0923+392$ has a flat spectrum at $\lambda \leq 2.8 \mathrm{~cm}$

- through the whole range of observing wavelengths, sources $0016+731,0235+164,0716+714,0804+499$, $0954+658,1039+811,1150+812,1652+398,1739+522$, $1749+701,1823+568$ and $2200+420$ have flat spectra. It is further noticeable that most of the steep spectrum sources are not variable with an exceptionally weak variability for 3C 309.1. In other words, variability is a common phenomenon in the flat spectrum sources. This is consistent with previous evidence that most variable sources have flat spectra (Kellermann 1974; Fanti et al. 1981).

We also investigate variations of the spectral indices, by calculating a two-point power law spectrum $(6 \mathrm{~cm}$ and $2.8 \mathrm{~cm}$, simply derived from $\left.\alpha_{2.8}^{6}=\frac{\ln \left(S_{6} / S_{2.8}\right)}{\ln \left(\lambda_{6} / \lambda_{2.8}\right)}\right)$, for those sources showing correspondingly at least one pronounced outburst at both wavelengths with enough simultaneous
Table 2. Summary of source variabilities at $21 \mathrm{~cm} . N$ denotes number of observations during this 5.5 years period, $\langle S\rangle$ the mean flux in Jy, $m$ the modulation index in $\%$ and $\chi_{\text {red }}^{2}$ the reduced $\chi^{2}$ value (we denote $>$ when the $\chi_{\text {red }}^{2} \geq 100.0$ ). For the statistical analysis ( $m$ and $\chi_{\text {red }}^{2}$ ), only data sets with $N \geq$ 8 are taken into consideration

\begin{tabular}{|c|c|c|c|c|}
\hline Source & $N$ & $\langle S\rangle[\mathrm{Jy}]$ & $m[\%]$ & $\chi_{\mathrm{red}}^{2}$ \\
\hline $0134+329$ & 6 & $16.14 \pm 0.21$ & & \\
\hline $0153+744$ & 5 & $1.95 \pm 0.05$ & & \\
\hline $0212+735$ & 5 & $2.38 \pm 0.03$ & & \\
\hline $0316+413$ & 4 & $27.28 \pm 0.27$ & & \\
\hline $0420-014$ & 2 & $2.08 \pm 0.10$ & & \\
\hline $0454+844$ & 6 & $0.28 \pm 0.05$ & & \\
\hline $0518+165$ & 6 & $8.65 \pm 0.09$ & & \\
\hline $0528+134$ & 4 & $2.46 \pm 0.34$ & & \\
\hline $0538+498$ & 4 & $21.76 \pm 0.14$ & & \\
\hline $0615+820$ & 5 & $0.69 \pm 0.02$ & & \\
\hline $0716+714$ & 7 & $0.78 \pm 0.07$ & & \\
\hline $0804+499$ & 4 & $0.97 \pm 0.08$ & & \\
\hline $0835+580$ & 5 & $2.26 \pm 0.02$ & & \\
\hline $0836+710$ & 7 & $3.47 \pm 0.18$ & & \\
\hline $0851+202$ & 2 & $1.14 \pm 0.07$ & & \\
\hline $0917+624$ & 5 & $1.24 \pm 0.06$ & & \\
\hline $0954+658$ & 5 & $0.48 \pm 0.04$ & & \\
\hline $1039+811$ & 5 & $0.76 \pm 0.02$ & & \\
\hline $1150+812$ & 5 & $1.64 \pm 0.07$ & & \\
\hline $1226+023$ & 1 & $51.88 \pm 0.52$ & & \\
\hline $1253-055$ & 1 & $9.32 \pm 0.09$ & & \\
\hline $1328+307$ & 6 & $14.65 \pm 0.05$ & & \\
\hline $1409+524$ & 6 & $22.14 \pm 0.11$ & & \\
\hline $1641+399$ & 4 & $7.54 \pm 0.15$ & & \\
\hline $1652+398$ & 3 & $1.82 \pm 0.04$ & & \\
\hline $1739+522$ & 3 & $1.43 \pm 0.42$ & & \\
\hline $1749+701$ & 5 & $0.79 \pm 0.02$ & & \\
\hline $1803+784$ & 7 & $2.07 \pm 0.17$ & & \\
\hline $1823+568$ & 3 & $1.56 \pm 0.04$ & & \\
\hline $1928+738$ & 5 & $3.95 \pm 0.05$ & & \\
\hline $2007+777$ & 6 & $0.95 \pm 0.16$ & & \\
\hline $2105+420$ & 4 & $1.39 \pm 0.03$ & & \\
\hline $2200+420$ & 3 & $4.39 \pm 0.34$ & & \\
\hline $2251+158$ & 2 & $14.35 \pm 0.74$ & & \\
\hline
\end{tabular}

measurements (with more than 8 data points) during the monitoring period. As seen from Figs. 26 and 27, the spectral indices $\left(\alpha_{2.8}^{6}\right)$ follow the expectations of shock models (Marscher \& Gear 1985; Valtaoja et al. 1992; Qian 1996) that shocks propagate from an optically thick to an optically thin regime in each outburst, most evidently seen in sources $0235+164,0528+165,0716+714,0804+499$, $0836+710,1641+399$ and $2007+777$, so that the variations in these sources were probably intrinsic.

\subsection{Statistical analysis}

We measure the degree of variability by deriving the modulation index $m[\%]=100 \times \frac{\sigma_{S}}{\langle S\rangle}$, where $<>$ denotes the mean. To see whether a source is variable, we performed a 
Table 3. Summary of source variability at $18 \mathrm{~cm}$

\begin{tabular}{|c|c|c|c|c|}
\hline Source & $N$ & $\langle S\rangle[\mathrm{Jy}]$ & $m[\%]$ & $\chi_{\mathrm{red}}^{2}$ \\
\hline $0134+329$ & 6 & $14.00 \pm 0.19$ & & \\
\hline $0153+744$ & 2 & $2.02 \pm 0.01$ & & \\
\hline $0212+735$ & 3 & $2.33 \pm 0.06$ & & \\
\hline $0316+413$ & 7 & $28.79 \pm 0.68$ & & \\
\hline 0420-014 & 2 & $2.22 \pm 0.30$ & & \\
\hline $0454+844$ & 2 & $0.25 \pm 0.03$ & & \\
\hline $0518+165$ & 6 & $7.76 \pm 0.14$ & & \\
\hline $0528+134$ & 3 & $2.21 \pm 0.05$ & & \\
\hline $0538+498$ & 4 & $19.79 \pm 0.19$ & & \\
\hline $0615+820$ & 2 & $0.75 \pm 0.02$ & & \\
\hline $0716+714$ & 5 & $0.78 \pm 0.11$ & & \\
\hline $0804+499$ & 3 & $0.92 \pm 0.05$ & & \\
\hline $0835+580$ & 2 & $1.93 \pm 0.03$ & & \\
\hline $0836+710$ & 7 & $3.35 \pm 0.15$ & & \\
\hline $0851+202$ & 3 & $1.41 \pm 0.26$ & & \\
\hline $0917+624$ & 8 & $1.27 \pm 0.06$ & 4.6 & 5.0 \\
\hline $0954+658$ & 4 & $0.48 \pm 0.13$ & & \\
\hline $1039+811$ & 2 & $0.73 \pm 0.04$ & & \\
\hline $1150+812$ & 2 & $1.61 \pm 0.13$ & & \\
\hline $1226+023$ & 7 & $49.30 \pm 1.77$ & & \\
\hline $1253-055$ & 5 & $10.01 \pm 0.31$ & & \\
\hline $1328+307$ & 10 & $13.53 \pm 0.11$ & 0.9 & 0.2 \\
\hline $1409+524$ & 9 & $19.27 \pm 0.18$ & 1.0 & 0.3 \\
\hline $1641+399$ & 5 & $7.46 \pm 0.38$ & & \\
\hline $1652+398$ & 1 & $1.80 \pm 0.02$ & & \\
\hline $1749+701$ & 2 & $0.76 \pm 0.02$ & & \\
\hline $1803+784$ & 5 & $2.25 \pm 0.03$ & & \\
\hline $1928+738$ & 8 & $3.92 \pm 0.15$ & 4.0 & 6.2 \\
\hline $2007+777$ & 4 & $1.01 \pm 0.19$ & & \\
\hline $2105+420$ & 7 & $1.87 \pm 0.03$ & & \\
\hline $2200+420$ & 2 & $3.18 \pm 1.10$ & & \\
\hline $2251+158$ & 4 & $14.27 \pm 0.36$ & & \\
\hline
\end{tabular}

$\chi^{2}$ test, $\chi^{2} \equiv \sum\left(\frac{S_{i}-<S>}{\sigma_{i}^{2}}\right)^{2}$, (similarly to, e.g. Fanti et al. 1981 and Bondi et al. 1996), where the uncertainties $\sigma_{i}$ are a combination of the experimental uncertainties $\sigma_{\mathrm{e} i}$ and the statistical uncertainties $\sigma_{\mathrm{s} i}$ at the $i$-th epoch, i.e., $\sigma_{i}^{2}=\sigma_{\mathrm{e} i}^{2}+\sigma_{\mathrm{s} i}^{2}$ (Eqs. (6-1) in Bevington 1969).

Our data were not obtained under identical conditions but rather comprised various observations during such a long-term monitoring period. For each calibrator source, $\sigma_{\mathrm{s} i}$ is taken to be the standard deviation of the data set during the monitoring period, and for each target source, $\sigma_{\mathrm{s} i}$ is approximated by taking the value of the $m_{0, \max } \times<S>$, where $m_{0, \max }$ is the maximum of the $m_{0}$ (the modulation index of a non-variable source) from calibrators at each wavelength. We take $m_{0, \max }$ as $1.0 \%$ at $\lambda \geq 11 \mathrm{~cm}$, as $1.2,2.0,2.3$ and $3.1 \%$ at $\lambda=6,3.6,2.8$ and $1.3 \mathrm{~cm}$, and as $4.6 \%$ at $\lambda \leq 9 \mathrm{~mm}$ respectively.

The statistical results (only for data sets with $N \geq 8$ at each wavelength) are presented in Tables $2-11$ for all sources at wavelengths of $21,18,11,6,3.6,2.8,2$ and $1.3 \mathrm{~cm}, 9$ and $7 \mathrm{~mm}$ respectively, with the source name, number of observations, the mean flux density with esti-
Table 4. Summary of source variabilities at $11 \mathrm{~cm}$. In the last column we denote $>$ when the $\chi_{\text {red }}^{2} \geq 100.0$

\begin{tabular}{crrrr}
\hline Source & $N$ & $<S>[\mathrm{Jy}]$ & $m[\%]$ & $\chi_{\text {red }}^{2}$ \\
\hline $0016+731$ & 3 & $1.35 \pm 0.58$ & & \\
$0134+329$ & 24 & $9.44 \pm 0.09$ & 0.9 & 0.5 \\
$0153+744$ & 2 & $1.86 \pm 0.02$ & & \\
$0212+735$ & 3 & $2.64 \pm 0.24$ & & \\
$0235+164$ & 8 & $1.31 \pm 0.56$ & 45.7 & $>$ \\
$0316+413$ & 7 & $30.37 \pm 2.46$ & & \\
$0420-014$ & 7 & $2.35 \pm 0.08$ & & \\
$0454+844$ & 15 & $0.31 \pm 0.06$ & 19.4 & $>$ \\
$0518+165$ & 12 & $5.71 \pm 0.03$ & 0.5 & 0.3 \\
$0528+134$ & 15 & $2.76 \pm 0.23$ & 8.5 & 26.8 \\
$0538+498$ & 4 & $13.34 \pm 0.04$ & & \\
$0615+820$ & 2 & $0.87 \pm 0.03$ & & \\
$0716+714$ & 43 & $0.64 \pm 0.16$ & 25.1 & $>$ \\
$0735+178$ & 2 & $2.28 \pm 0.01$ & & \\
$0804+499$ & 15 & $1.02 \pm 0.11$ & 10.6 & 47.1 \\
$0835+580$ & 8 & $1.15 \pm 0.01$ & 0.4 & 0.2 \\
$0836+710$ & 39 & $2.60 \pm 0.16$ & 6.2 & 22.3 \\
$0851+202$ & 17 & $1.38 \pm 0.18$ & 13.2 & 94.0 \\
$0917+624$ & 15 & $1.40 \pm 0.12$ & 8.5 & 17.7 \\
$0923+392$ & 15 & $6.01 \pm 0.20$ & 3.5 & 5.2 \\
$0951+699$ & 13 & $5.03 \pm 0.01$ & 0.3 & 0.1 \\
$0954+658$ & 20 & $0.48 \pm 0.11$ & 23.6 & $>$ \\
$1039+811$ & 3 & $0.79 \pm 0.07$ & & \\
$1150+812$ & 6 & $1.56 \pm 0.16$ & & \\
$1226+023$ & 4 & $45.20 \pm 1.79$ & & \\
$1328+307$ & 31 & $10.62 \pm 0.07$ & 0.7 & 0.3 \\
$1409+524$ & 20 & $12.28 \pm 0.11$ & 0.9 & 0.2 \\
$1458+718$ & 6 & $4.71 \pm 0.09$ & & \\
$1641+399$ & 7 & $8.34 \pm 0.22$ & & \\
$1652+398$ & 8 & $1.72 \pm 0.05$ & 3.1 & 3.8 \\
$1739+522$ & 9 & $1.37 \pm 0.51$ & 39.3 & $>$ \\
$1749+701$ & 4 & $0.71 \pm 0.04$ & & \\
$1803+784$ & 15 & $2.34 \pm 0.26$ & 11.5 & 66.6 \\
$1823+568$ & 1 & $1.43 \pm 0.01$ & & \\
$1928+738$ & 14 & $3.91 \pm 0.14$ & 3.6 & 6.9 \\
$2007+777$ & 13 & $1.36 \pm 0.15$ & 11.1 & 79.6 \\
$2105+420$ & 30 & $3.69 \pm 0.04$ & 1.0 & 0.7 \\
$2200+420$ & 14 & $3.71 \pm 0.63$ & 17.6 & $>$ \\
$2251+158$ & 5 & $13.77 \pm 0.46$ & & \\
\hline & & & &
\end{tabular}

mated error, the modulation index, and the reduced $\chi_{\text {red }}^{2}$. Obviously, the statistical results confirm that nearly all sources, with the exception of $0835+580$ and $0951+699$ and the six primary flux calibrators, are variable at a confidence level of more than $99.95 \%$ at most of the radio wavelengths over this 5.5 years period.

\subsection{Variability dependence}

We investigate whether there is any dependence of the degree of variability on either source galactic latitude $\left(b^{\mathrm{II}}\right)$, redshift $(z)$, spectral indices $\left(\alpha_{6}^{11}\right.$ and $\alpha_{2.8}^{6}$ only), or superluminal motions by taking the modulation index of each 
Table 5. Summary of source variabilities at $6 \mathrm{~cm}$. In the last column we denote $>$ when the $\chi_{\text {red }}^{2} \geq 100.0$

\begin{tabular}{crrrr}
\hline Source & $N$ & $\langle S\rangle[\mathrm{Jy}]$ & $m[\%]$ & $\chi_{\text {red }}^{2}$ \\
\hline $0016+731$ & 15 & $1.30 \pm 0.47$ & 37.5 & $>$ \\
$0134+329$ & 68 & $5.53 \pm 0.07$ & 1.2 & 0.6 \\
$0153+744$ & 12 & $1.21 \pm 0.04$ & 3.8 & 6.0 \\
$0212+735$ & 14 & $3.08 \pm 0.19$ & 6.5 & 19.3 \\
$0235+164$ & 19 & $1.19 \pm 0.84$ & 72.3 & $>$ \\
$0316+413$ & 29 & $25.71 \pm 3.19$ & 12.6 & 88.3 \\
$0420-014$ & 14 & $2.75 \pm 0.30$ & 11.5 & 51.9 \\
$0454+844$ & 31 & $0.32 \pm 0.05$ & 17.4 & $>$ \\
$0518+165$ & 35 & $3.79 \pm 0.04$ & 1.2 & 0.6 \\
$0528+134$ & 37 & $4.29 \pm 1.31$ & 30.9 & $>$ \\
$0538+498$ & 13 & $7.92 \pm 0.08$ & 1.0 & 0.5 \\
$0615+820$ & 10 & $0.77 \pm 0.03$ & 4.2 & 6.0 \\
$0716+714$ & 74 & $0.66 \pm 0.25$ & 37.7 & $>$ \\
$0735+178$ & 8 & $1.91 \pm 0.33$ & 18.3 & $>$ \\
$0804+499$ & 35 & $1.00 \pm 0.28$ & 28.4 & $>$ \\
$0835+580$ & 30 & $0.60 \pm 0.01$ & 1.0 & 0.4 \\
$0836+710$ & 75 & $2.12 \pm 0.21$ & 10.2 & 45.2 \\
$0851+202$ & 33 & $1.62 \pm 0.24$ & 15.0 & 78.0 \\
$0917+624$ & 45 & $1.53 \pm 0.09$ & 5.8 & 10.1 \\
$0923+392$ & 29 & $10.98 \pm 0.35$ & 3.3 & 4.2 \\
$0951+699$ & 32 & $3.33 \pm 0.03$ & 0.8 & 0.3 \\
$0954+658$ & 39 & $0.50 \pm 0.13$ & 26.8 & $>$ \\
$1039+811$ & 12 & $0.81 \pm 0.06$ & 7.9 & 22.6 \\
$1150+812$ & 14 & $1.50 \pm 0.11$ & 7.9 & 20.3 \\
$1226+023$ & 20 & $39.48 \pm 2.32$ & 6.0 & 11.8 \\
$1253-055$ & 11 & $14.40 \pm 2.82$ & 20.5 & $>$ \\
$1328+307$ & 74 & $7.49 \pm 0.07$ & 0.9 & 0.7 \\
$1409+524$ & 39 & $6.58 \pm 0.08$ & 1.1 & 0.7 \\
$1458+718$ & 10 & $3.36 \pm 0.16$ & 5.1 & 9.6 \\
$1641+399$ & 27 & $8.47 \pm 0.29$ & 3.4 & 3.8 \\
$1652+398$ & 13 & $1.59 \pm 0.04$ & 2.4 & 2.2 \\
$1739+522$ & 21 & $1.48 \pm 0.48$ & 33.1 & $>$ \\
$1749+701$ & 14 & $0.69 \pm 0.06$ & 8.6 & 22.2 \\
$1803+784$ & 40 & $2.60 \pm 0.23$ & 9.1 & 31.8 \\
$1823+568$ & 6 & $1.64 \pm 0.20$ & & \\
$1928+738$ & 36 & $3.92 \pm 0.16$ & 4.2 & 6.8 \\
$2007+777$ & 34 & $1.39 \pm 0.22$ & 16.4 & 96.1 \\
$2105+420$ & 66 & $5.48 \pm 0.06$ & 1.0 & 0.5 \\
$2200+420$ & 23 & $3.90 \pm 0.89$ & 23.3 & $>$ \\
$2251+158$ & 26 & $14.97 \pm 1.65$ & 11.3 & 49.4 \\
\hline & & & &
\end{tabular}

source at $6 \mathrm{~cm}$, where the sources have been observed most frequently.

As shown in Fig. 28, there is no obvious dependence of the degree of variability on most of the above mentioned quantities. This is confirmed by the formal estimates of the correlation probabilities (coefficients are less than 0.26 ) of these relationships with an exception, that is a weak correlation (coefficient is 0.48 ) between the degree of variability and the 6 to $2.8 \mathrm{~cm}$ spectral index. Both the degree of variability and its rate of occurrence appear to be higher when the source spectra are flatter. This is consistent with previous findings: sources with flat spectra are small, and are variable, while sources with steep spec-
Table 6. Summary of source variability at $3.6 \mathrm{~cm}$. In the last column we denote $>$ when the $\chi_{\text {red }}^{2} \geq 100.0$

\begin{tabular}{crrcr}
\hline Source & $N$ & $<S>[\mathrm{Jy}]$ & $m[\%]$ & $\chi_{\text {red }}^{2}$ \\
\hline $0016+731$ & 6 & $1.39 \pm 0.40$ & & \\
$0134+329$ & 9 & $3.22 \pm 0.04$ & 1.2 & 0.4 \\
$0153+744$ & 1 & $0.64 \pm 0.01$ & & \\
$0212+735$ & 1 & $3.08 \pm 0.04$ & & \\
$0235+164$ & 2 & $2.48 \pm 1.67$ & & \\
$0316+413$ & 21 & $24.53 \pm 2.39$ & 10.0 & 14.0 \\
$0420-014$ & 1 & $3.00 \pm 0.15$ & & \\
$0454+844$ & 5 & $0.29 \pm 0.06$ & & \\
$0518+165$ & 6 & $2.37 \pm 0.07$ & & \\
$0528+134$ & 7 & $3.49 \pm 0.70$ & & \\
$0538+498$ & 5 & $4.67 \pm 0.19$ & & \\
$0615+820$ & 1 & $0.64 \pm 0.01$ & & \\
$0716+714$ & 16 & $0.82 \pm 0.35$ & 44.4 & $>$ \\
$0735+178$ & 1 & $2.15 \pm 0.03$ & & \\
$0804+499$ & 6 & $1.36 \pm 0.46$ & & \\
$0835+580$ & 5 & $0.31 \pm 0.01$ & & \\
$0836+710$ & 19 & $1.84 \pm 0.41$ & 22.9 & 77.5 \\
$0851+202$ & 8 & $2.01 \pm 0.26$ & 13.9 & 30.0 \\
$0917+624$ & 14 & $1.71 \pm 0.21$ & 12.7 & 29.5 \\
$0923+392$ & 11 & $13.29 \pm 0.41$ & 3.2 & 1.1 \\
$0951+699$ & 4 & $2.12 \pm 0.03$ & & \\
$0954+658$ & 8 & $0.45 \pm 0.16$ & 38.5 & $>$ \\
$1039+811$ & 2 & $0.95 \pm 0.24$ & & \\
$1226+023$ & 5 & $38.79 \pm 3.14$ & & \\
$1253-055$ & 6 & $14.66 \pm 0.57$ & & \\
$1328+307$ & 20 & $5.11 \pm 0.07$ & 1.5 & 0.4 \\
$1409+524$ & 11 & $3.38 \pm 0.04$ & 1.2 & 0.2 \\
$1458+718$ & 2 & $2.55 \pm 0.04$ & & \\
$1641+399$ & 11 & $8.45 \pm 0.44$ & 5.4 & 4.3 \\
$1652+398$ & 3 & $1.53 \pm 0.04$ & & \\
$1739+522$ & 4 & $1.20 \pm 0.62$ & & \\
$1749+701$ & 3 & $0.59 \pm 0.05$ & & \\
$1803+784$ & 11 & $2.51 \pm 0.23$ & 9.5 & 12.8 \\
$1928+738$ & 5 & $3.71 \pm 0.22$ & & \\
$2007+777$ & 4 & $1.31 \pm 0.13$ & & \\
$2105+420$ & 12 & $6.02 \pm 0.12$ & 2.0 & 0.3 \\
$2200+420$ & 7 & $3.17 \pm 0.64$ & & \\
$2251+158$ & 3 & $13.91 \pm 1.05$ & & \\
\hline & & & &
\end{tabular}

tra are less variable (Heeschen et al. 1987). Furthermore, there is a trend showing that the degree of variability decreases with increasing wavelength, as seen in Fig. 29 by taking only the derived $m$ of the common 16 sources at the four wavelengths of $11,6,2.8$ and $1.3 \mathrm{~cm}$, which has been shown in other variability studies (e.g. Peng et al. 2000). The maximum values for $m$ are derived to be 25.1 , $37.7,54.0$ and $60.9 \%$ respectively at the four wavelengths sequenced above. This is contrary to the expectations of the interstellar scintillation (ISS, e.g. Rickett 1990), but in favor of shock models (e.g. Marscher \& Gear 1985). 
Table 7. Summary of source variabilities at $2.8 \mathrm{~cm}$. In the last column we denote $>$ when the $\chi_{\text {red }}^{2} \geq 100.0$

\begin{tabular}{crrrr}
\hline Source & $N$ & $<S>[\mathrm{Jy}]$ & $m[\%]$ & $\chi_{\text {red }}^{2}$ \\
\hline $0016+731$ & 5 & $1.37 \pm 0.41$ & & \\
$0134+329$ & 37 & $2.58 \pm 0.05$ & 2.1 & 0.6 \\
$0153+744$ & 7 & $0.58 \pm 0.01$ & & \\
$0212+735$ & 13 & $3.18 \pm 0.28$ & 9.1 & 10.1 \\
$0235+164$ & 15 & $1.83 \pm 1.29$ & 73.0 & $>$ \\
$0316+413$ & 13 & $22.23 \pm 1.13$ & 5.3 & 3.3 \\
$0420-014$ & 14 & $3.31 \pm 0.30$ & 9.3 & 12.7 \\
$0454+844$ & 22 & $0.28 \pm 0.06$ & 21.1 & 49.1 \\
$0518+165$ & 28 & $2.08 \pm 0.05$ & 2.3 & 0.5 \\
$0528+134$ & 29 & $4.84 \pm 1.96$ & 41.3 & $>$ \\
$0538+498$ & 6 & $3.95 \pm 0.08$ & & \\
$0615+820$ & 5 & $0.58 \pm 0.04$ & & \\
$0716+714$ & 59 & $0.73 \pm 0.39$ & 54.0 & $>$ \\
$0735+178$ & 3 & $1.23 \pm 0.30$ & & \\
$0804+499$ & 23 & $1.10 \pm 0.50$ & 46.4 & $>$ \\
$0835+580$ & 13 & $0.24 \pm 0.01$ & 2.0 & 0.5 \\
$0836+710$ & 58 & $1.91 \pm 0.52$ & 27.3 & $>$ \\
$0851+202$ & 21 & $1.82 \pm 0.35$ & 19.9 & 45.9 \\
$0917+624$ & 29 & $1.51 \pm 0.16$ & 10.8 & 9.4 \\
$0923+392$ & 20 & $13.11 \pm 0.42$ & 3.3 & 1.5 \\
$0951+699$ & 12 & $1.87 \pm 0.03$ & 1.8 & 0.4 \\
$0954+658$ & 22 & $0.52 \pm 0.14$ & 28.4 & 90.6 \\
$1039+811$ & 11 & $1.11 \pm 0.08$ & 7.4 & 7.8 \\
$1150+812$ & 4 & $1.51 \pm 0.06$ & & \\
$1226+023$ & 6 & $34.09 \pm 4.03$ & & \\
$1253-055$ & 3 & $23.14 \pm 6.02$ & & \\
$1328+307$ & 44 & $4.45 \pm 0.06$ & 1.3 & 0.3 \\
$1409+524$ & 31 & $2.60 \pm 0.03$ & 1.1 & 0.6 \\
$1458+718$ & 12 & $2.34 \pm 0.17$ & 7.6 & 8.4 \\
$1641+399$ & 15 & $7.96 \pm 0.60$ & 7.8 & 5.4 \\
$1652+398$ & 13 & $1.40 \pm 0.04$ & 2.7 & 0.9 \\
$1739+522$ & 14 & $1.52 \pm 0.63$ & 43.1 & $>$ \\
$1749+701$ & 4 & $0.66 \pm 0.10$ & & \\
$1803+784$ & 25 & $2.70 \pm 0.20$ & 7.4 & 7.1 \\
$1823+568$ & 8 & $1.67 \pm 0.33$ & 21.0 & 62.4 \\
$1928+738$ & 17 & $3.76 \pm 0.25$ & 6.7 & 4.1 \\
$2007+777$ & 23 & $1.34 \pm 0.17$ & 13.2 & 22.0 \\
$2105+420$ & 44 & $6.05 \pm 0.10$ & 1.7 & 0.5 \\
$2200+420$ & 20 & $4.02 \pm 0.63$ & 16.1 & 28.8 \\
$2251+158$ & 15 & $12.03 \pm 2.04$ & 17.6 & 48.1 \\
\hline & & & &
\end{tabular}

Table 8. Summary of source variability at $2 \mathrm{~cm}$

\begin{tabular}{crrrc}
\hline Source & $N$ & $\langle S\rangle[\mathrm{Jy}]$ & $m[\%]$ & $\chi_{\text {red }}^{2}$ \\
\hline $0538+498$ & 4 & $2.88 \pm 0.14$ & & \\
$1409+524$ & 7 & $1.69 \pm 0.05$ & & \\
$1641+399$ & 6 & $7.73 \pm 0.52$ & & \\
$2105+420$ & 6 & $5.82 \pm 0.13$ & & \\
\hline
\end{tabular}

Table 9. Summary of source variabilities at $1.3 \mathrm{~cm}$

\begin{tabular}{|c|c|c|c|c|}
\hline Source & $N$ & $\langle S\rangle$ [Jy] & $m[\%]$ & $\chi_{\mathrm{red}}^{2}$ \\
\hline $0016+731$ & 2 & $0.66 \pm 0.19$ & & \\
\hline $0134+329$ & 10 & $1.11 \pm 0.03$ & 2.8 & 0.4 \\
\hline $0153+744$ & 2 & $0.42 \pm 0.02$ & & \\
\hline $0212+735$ & 3 & $2.25 \pm 0.28$ & & \\
\hline $0235+164$ & 4 & $2.04 \pm 1.78$ & & \\
\hline $0316+413$ & 12 & $17.23 \pm 1.89$ & 11.4 & 5.8 \\
\hline 0420-014 & 9 & $3.57 \pm 0.44$ & 12.9 & 3.4 \\
\hline $0454+844$ & 6 & $0.33 \pm 0.13$ & & \\
\hline $0518+165$ & 10 & $1.10 \pm 0.03$ & 3.1 & 0.3 \\
\hline $0528+134$ & 14 & $4.17 \pm 1.58$ & 39.3 & 49.5 \\
\hline $0538+498$ & 5 & $1.91 \pm 0.11$ & & \\
\hline $0615+820$ & 1 & $0.40 \pm 0.02$ & & \\
\hline $0716+714$ & 19 & $0.92 \pm 0.55$ & 60.9 & 92.3 \\
\hline $0804+499$ & 8 & $1.10 \pm 0.33$ & 32.5 & 30.1 \\
\hline $0836+710$ & 49 & $1.74 \pm 0.38$ & 22.2 & 23.9 \\
\hline $0851+202$ & 10 & $1.92 \pm 0.30$ & 16.3 & 9.8 \\
\hline $0917+624$ & 10 & $1.19 \pm 0.09$ & 7.5 & 3.2 \\
\hline $0923+392$ & 5 & $11.13 \pm 0.62$ & & \\
\hline $0951+699$ & 2 & $1.00 \pm 0.06$ & & \\
\hline $0954+658$ & 7 & $0.55 \pm 0.19$ & & \\
\hline $1150+812$ & 1 & $1.06 \pm 0.06$ & & \\
\hline $1226+023$ & 3 & $34.27 \pm 9.55$ & & \\
\hline $1328+307$ & 16 & $2.46 \pm 0.05$ & 1.9 & 0.2 \\
\hline $1409+524$ & 12 & $0.92 \pm 0.02$ & 2.8 & 0.3 \\
\hline $1458+718$ & 2 & $1.42 \pm 0.22$ & & \\
\hline $1641+399$ & 10 & $7.45 \pm 0.75$ & 10.7 & 5.4 \\
\hline $1652+398$ & 8 & $1.13 \pm 0.07$ & 6.5 & 1.6 \\
\hline $1739+522$ & 6 & $1.64 \pm 0.64$ & & \\
\hline $1749+701$ & 1 & $0.62 \pm 0.05$ & & \\
\hline $1803+784$ & 12 & $2.27 \pm 0.12$ & 5.6 & 1.5 \\
\hline $1928+738$ & 9 & $3.27 \pm 0.27$ & 8.8 & 3.0 \\
\hline $2007+777$ & 12 & $1.16 \pm 0.10$ & 9.0 & 3.0 \\
\hline $2105+420$ & 22 & $5.58 \pm 0.10$ & 1.8 & 0.2 \\
\hline $2200+420$ & 8 & $4.00 \pm 0.62$ & 16.5 & 10.6 \\
\hline $2251+158$ & 6 & $10.26 \pm 3.44$ & & \\
\hline
\end{tabular}

Table 10. Summary of source variability at $9 \mathrm{~mm}$

\begin{tabular}{crcrr}
\hline Source & $N$ & $\langle S\rangle[\mathrm{Jy}]$ & $m[\%]$ & $\chi_{\text {red }}^{2}$ \\
\hline $0134+329$ & 10 & $0.81 \pm 0.02$ & 2.2 & 0.3 \\
$0518+165$ & 6 & $0.76 \pm 0.07$ & & \\
$0716+714$ & 21 & $0.88 \pm 0.53$ & 60.9 & 54.5 \\
$0851+202$ & 4 & $2.03 \pm 0.37$ & & \\
$0917+624$ & 10 & $1.01 \pm 0.07$ & 6.9 & 0.6 \\
$0954+658$ & 5 & $0.45 \pm 0.19$ & & \\
$1328+307$ & 15 & $1.92 \pm 0.03$ & 1.7 & 0.1 \\
$1409+524$ & 10 & $0.56 \pm 0.02$ & 4.6 & 0.3 \\
$1652+398$ & 4 & $1.00 \pm 0.03$ & & \\
$1739+522$ & 4 & $1.57 \pm 0.51$ & & \\
$1928+738$ & 4 & $2.73 \pm 0.29$ & & \\
$2007+777$ & 8 & $1.05 \pm 0.16$ & 16.0 & 3.5 \\
$2105+420$ & 15 & $5.25 \pm 0.14$ & 2.7 & 0.3 \\
$2200+420$ & 11 & $3.61 \pm 0.58$ & 16.8 & 7.0 \\
$2251+158$ & 3 & $8.01 \pm 1.14$ & & \\
\hline
\end{tabular}


Table 11. Summary of source variability at $7 \mathrm{~mm}$

\begin{tabular}{crrcc}
\hline Source & $N$ & $\langle S\rangle[\mathrm{Jy}]$ & $m[\%]$ & $\chi_{\text {red }}^{2}$ \\
\hline $0134+329$ & 7 & $0.55 \pm 0.07$ & & \\
$0316+413$ & 4 & $11.87 \pm 2.51$ & & \\
$0518+165$ & 5 & $0.63 \pm 0.05$ & & \\
$0528+134$ & 7 & $4.39 \pm 1.76$ & & \\
$0716+714$ & 6 & $0.92 \pm 0.53$ & & \\
$0804+499$ & 5 & $1.08 \pm 0.30$ & & \\
$0836+710$ & 25 & $1.63 \pm 0.32$ & 20.2 & 7.7 \\
$0851+202$ & 5 & $1.52 \pm 0.35$ & & \\
$1328+307$ & 7 & $1.49 \pm 0.03$ & & \\
$1409+524$ & 5 & $0.44 \pm 0.08$ & & \\
$1641+399$ & 6 & $6.79 \pm 0.74$ & & \\
$1803+784$ & 5 & $1.89 \pm 0.27$ & & \\
$1928+738$ & 4 & $2.86 \pm 0.32$ & & \\
$2105+420$ & 10 & $5.01 \pm 0.22$ & 4.6 & 0.3 \\
\hline
\end{tabular}

\section{Comments on individual sources}

In the context, the apparent brightness temperature is given by Gopal-Krishna et al. (1984),

$T_{\text {app }} \geq \frac{2 c^{2} \triangle S}{\pi \kappa \nu^{2} \theta_{\text {app }}^{2}} \mathrm{~K}$,

when transforming to the co-moving frame of the source it becomes,

$T_{\text {app }}^{\prime} \geq T_{\text {app }}(1+z)^{3} \delta^{-3} \mathrm{~K}$,

where $\triangle S$ is the flux change during a period $\tau, \theta_{\text {app }} \leq$ $2 c \tau / D_{\mathrm{a}}$ the apparent diameter of the variable component based on the causality argument, $D_{\mathrm{a}}$ (Lang 1974) the angular distance of the source, and $\delta$ the Doppler factor.

- 0016+734: This quasar $(z=1.781$, in which highly superluminal motion is observed, SLM hereafter) shows a monotonic flux decrease at $6,3.6$ and $2.8 \mathrm{~cm}$. Its modulation index is $38 \%$ at $6 \mathrm{~cm}$. It belongs to a complete sample of flat spectrum sources in the S5 survey (S5 sample hereafter);

- 0134+329 (3C 48): This quasar $(z=0.367)$ shows no flux variations as expected. It has been used as a primary flux calibrator;

- 0153+744: In this quasar $(z=2.338$, showing highly SLM), the modulation index is $3.8 \%$ at $6 \mathrm{~cm}$. It belongs to the S5 sample;

- 0212+735: In this quasar $(z=2.367$, highly SLM), the modulation index increases with decreasing wavelength from $6.5 \%$ at $6 \mathrm{~cm}$ to $9.1 \%$ at $2.8 \mathrm{~cm}$. Its spectrum at 6 and $2.8 \mathrm{~cm}$ has been inverted since around late 1996. It belongs to the S5 sample;

- 0235+164: In this BL Lac object $(z=0.940$, highly SLM), a pronounced outburst started at early 1997 and lasted to the end of our monitoring period at all observing wavelengths. Its modulation index increases with decreasing wavelength at 11, 6 and $2.8 \mathrm{~cm}$. It is a IDV source discussed recently by Kraus et al (1999a). Its angular-diameter distance $D_{\mathrm{a}}$ is $1.7210^{3} \mathrm{Mpc}$. Because of the flux change $\triangle S=2.88 \mathrm{Jy}$ at $2.8 \mathrm{~cm}$ during $\tau \sim 229$ days (which corresponds to the fastest flux change in the lightcurve), this corresponds to an apparent brightness temperature in the co-moving frame of the quasar, $T_{\mathrm{app}}^{\prime} \geq 1.5910^{14} \delta^{-3} \mathrm{~K}$. If the large excess of the brightness temperature over the inverse Compton limit of $10^{12} \mathrm{~K}$ (Kellermann \& Pauliny-Toth 1969 ) is due to a relativistic bulk motion (as suggested by Rees $1966 \mathrm{~b}$ and by Woltjer 1966) of the variable component, a Doppler factor $\delta \geq 5.4$ is needed. The resultant $\delta$ is well within the "canonical" value of 10 , derived from VLBI and X-ray observations (see Ghisellini et al. 1993; Zensus 1997; Wagner \& Witzel 1995);

- 0316+413 (3C 84): This galaxy $(z=0.017$, showing subluminal motion, Sub-LM hereafter) mostly shows a slow, monotonic flux decrease (with an exception at $21 \mathrm{~cm}$ where it is not variable), but with an increase in the middle of 1998 at $3.6 \mathrm{~cm}$ and a significant increase at $7 \mathrm{~mm}$ since 1994. Its modulation index at the level of $5-13 \%$ varies irregularly with wavelength;

- 0420-014: In this quasar $(z=0.915)$, two outbursts cover the whole monitoring period at all observing wavelengths. Its modulation index decreases firstly from $11.5 \%$ at $6 \mathrm{~cm}$ to $9.3 \%$ at $2.8 \mathrm{~cm}$, then increases to $12.9 \%$ at $1.3 \mathrm{~cm}$

- 0454+844: This BL Lac object $(z=0.112$, SLM), shows firstly a monotonic flux increase till the middle of 1995, then a monotonic decrease. Its modulation index at the level of $17-21 \%$ varies up and down with decreasing wavelength. It belongs to the S5 sample;

- 0518+165 (3C 138): This quasar $(z=0.759)$ shows no flux variations as expected. It has been used as a primary flux calibrator;

- 0528+134: In this quasar $(z=2.060$, SLM), two pronounced outbursts covered the whole monitoring period at most of the observing wavelengths, but measurements were not available at 11 and $1.3 \mathrm{~cm}$ during the outbursts. The modulation index increases with decreasing wavelength at 11,6 and $2.8 \mathrm{~cm}$, but decreases by about $2 \%$ at $1.3 \mathrm{~cm}$. The angular distance $D_{\text {a }}$ is $1.6310^{3} \mathrm{Mpc}$. The flux change $\triangle S=2.18 \mathrm{Jy}$ at $6 \mathrm{~cm}$ during $\tau \sim 167.8$ days (the fastest flux change in the lightcurve) corresponds to an apparent brightness temperature $T_{\text {app }}^{\prime} \geq 3.4810^{15} \delta^{-3} \mathrm{~K}$ in the comoving frame of the quasar. A Doppler factor $\delta \geq 15$ is needed in terms of a relativistic bulk motion, which is higher than the "canonical" value of 10 . However this agrees well with the suggested Doppler boosting (Krichbaum et al. 1995) and the observed superluminal motion (Krichbaum et al. 1995; Britzen et al. 1999) in this source, which is one of the strongest gamma-active blazars (Britzen et al. 1999);

- 0538+498 (3C 147): This quasar $(z=0.545)$ shows no flux variations as expected. It has been used as a primary flux calibrator; 
- 0615+820: In this quasar $(z=0.710, \mathrm{SLM})$, the modulation index is $4.2 \%$ at $6 \mathrm{~cm}$. It belongs to the $\mathrm{S} 5$ sample;

- 0716+714: In this BL Lac object (at uncertain redshift $z=0.30$, SLM), (quasi-)periodic flux variations appear simultaneously at all observing wavelengths. Remarkably, its modulation index at the level of about $25-61 \%$ increases with decreasing wavelength, but has the same value at both $1.3 \mathrm{~cm}$ and $9 \mathrm{~mm}$. It belongs to the S5 sample, showing close radio-optical IDV correlations (Quirrenbach et al. 1992; Wagner et al. 1996). Rapid variations on time scales of a few hours have been observed at $5 \mathrm{GHz}$ (Quirrenbach et al. 1989). It is the only blazar showing the IDV phenomenon throughout the entire wavelength regime;

- 0735+178: In this BL Lac object $(z=0.424$, highly SLM), a monotonic flux decrease appears with a modulation index of $18.3 \%$ at $6 \mathrm{~cm}$;

- 0804+499: In this quasar $(z=1.433)$, a pronounced outburst started at early 1997 and continued to the end of the monitoring period at all observing wavelengths. Its modulation index increases by about $18 \%$ interval at wavelengths 11, 6 and $2.8 \mathrm{~cm}$, then decreases by about $14 \%$ at $1.3 \mathrm{~cm}$;

- 0835+580: This quasar $(z=1.534$, sub-SLM) shows no flux variations at all. Therefore it can be used as a secondary flux calibrator;

- 0836+710: In this quasar $(z=2.172$, highly SLM), a few outbursts appear with time delays at increasing wavelengths of $7 \mathrm{~mm}, 1.3,2.8$ and $6 \mathrm{~cm}$. Its modulation index increases with decreasing wavelength till $2.8 \mathrm{~cm}$, then decreases to about $20.2 \%$ at $7 \mathrm{~mm}$. VLBI monitoring observations during 1993-1996 revealed the ejection of a new jet component, and gamma to radio variations of the flux density were observed in the first half of 1992, indicating a correlation of the jet activity with the variability of the broad-band electromagnetic spectrum of the source (Otterbein et al. 1998). It belongs to the S5 sample, and once showed weak IDV at a very low level (Kraus 1997). No variations were seen by Quirrenbach et al. (2000), and it has been further suggested to serve as a polarization calibrator because of its high degree of polarization;

- 0851+202 (OJ287): In this BL Lac object $(z=0.306$, SLM), significant variations appear. Its modulation index increases with decreasing wavelength till $2.8 \mathrm{~cm}$, then decreases to $16.3 \%$ at $1.3 \mathrm{~cm}$;

- 0917+624: In this quasar $(z=1.446$, SLM), smooth variations appear. Its modulation index varies with wavelength. It is an IDV source with a change in the variability properties (Kraus et al. 1999b);

- 0923+392 (4C 39.25): In this quasar $(z=0.698, \mathrm{SLM})$, smooth flux variations appear with a wavelengthindependent modulation index of $3.3 \%$;
- 0951+699: This galaxy $(z=0.001)$ shows no flux variations at all. It can be used as a secondary flux calibrator;

- 0954+658: In this BL Lac object $(z=0.367)$, a pronounced outburst appears. Its modulation index increases with decreasing wavelength till $3.6 \mathrm{~cm}$, but decreases to $28.4 \%$ at $2.8 \mathrm{~cm}$;

- 1039+811: In this quasar $(z=1.254$, highly SLM), a flux increase appeared since early 1997 at 6 and $2.8 \mathrm{~cm}$. Its modulation index is almost the same at $6 \mathrm{~cm}$ and $2.8 \mathrm{~cm}$. It belongs to the $\mathrm{S} 5$ sample;

- 1150+812: In this quasar $(z=1.250$, SLM), a monotonic flux increase appears at 21,11 , and $6 \mathrm{~cm}$ since 1994. Its modulation index is $8 \%$ at $6 \mathrm{~cm}$. It belongs to the $\mathrm{S} 5$ sample;

$-1226+023$ (3C 273): In this quasar $(z=0.158$, highly SLM), smooth flux variations appear. Its modulation index is $6 \%$ at $6 \mathrm{~cm}$;

- 1253-055 (3C 279): In this quasar $(z=0.536$, highly SLM), smooth flux variation appears with a modulation index of $21 \%$ at $6 \mathrm{~cm}$.

$-1328+307$ (3C 286): This quasar $(z=0.849)$ shows no flux variations as expected. It has been used as a primary flux calibrator;

$-1409+524$ (3C 295): This galaxy $(z=0.461)$ shows no flux variations as expected. It has been used as a primary flux calibrator;

$-1458+718$ (3C 309.1): This quasar $(z=0.905$, highly SLM) has modulation indices of $5 \%$ at $6 \mathrm{~cm}$ and $7.6 \%$ at $2.8 \mathrm{~cm}$;

- 1641+399 (3C 345): In this quasar $(z=0.594$, highly SLM), smooth flux variations appear. Its modulation index increases with decreasing wavelength;

- 1652+398: In this BL Lac object $(z=0.034)$, very slow and weak flux variations appear. Its modulation index is $3 \%$ at $11 \mathrm{~cm}$, then increases with decreasing wavelength;

- 1739+522: In this quasar $(z=1.379)$, two (adjacent) continuous outbursts appear at 6 and $2.8 \mathrm{~cm}$. Its modulation index varies with wavelength;

- 1749+701: In this BL Lac object $(z=0.770$, SLM), a pronounced outburst appeared at $6 \mathrm{~cm}$ since mid1996. But there were no measurements available at other wavelengths during the outburst. Its modulation index is about $9 \%$ at $6 \mathrm{~cm}$. It belongs to the $\mathrm{S} 5$ sample;

- 1803+784: In this superluminal source, classified variously as a BL Lac object or quasar $(z=0.684)$, significant flux variations appear at $6 \mathrm{~cm}$. Its modulation index decreases from $11.5 \%$ to $5.6 \%$ between wavelengths of $11 \mathrm{~cm}$ and $1.3 \mathrm{~cm}$, but is almost the same at 6 and $3.6 \mathrm{~cm}$. It belongs to the $\mathrm{S} 5$ sample;

- 1823+568: In this BL Lac object $(z=0.664$, SLM), a monotonic flux decrease appears at both 6 and $2.8 \mathrm{~cm}$. Its modulation index is $21 \%$ at $2.8 \mathrm{~cm}$;

- 1928+738: In this quasar $(z=0.302$, highly SLM), weak flux fluctuations appeared at most wavelengths 
since 1994. Its modulation index mostly increases with decreasing wavelength. It belongs to the S5 sample, sometimes showing weak IDV (Heeschen et al. 1987; Kraus 1997), but no variations were seen by Quirrenbach et al. (2000);

$-2007+777$ : In this BL Lac object $(z=0.342$, SLM), outbursts appeared at wavelengths longer than $2.8 \mathrm{~cm}$. Its modulation index at the level of about $9-16 \%$ varies irregularly with wavelength. It belongs to the S5 sample. Simultaneous variability on time scales of a few days was found at radio, infrared and optical regimes (Peng et al. 2000);

- 2105+420 (NGC 7027): This planetary nebula (denote PN in Table 1), shows no flux variations as expected. It has been used as a primary flux calibrator;

$-2200+420$ : In this BL Lac object $(z=0.069$, SLM), a long-term (of more than 4 years at least) outburst on which many small flares are superimposed appeared. Its modulation index goes up by about $6 \%$ from 11 to $6 \mathrm{~cm}$, then appears almost wavelength-independent at shorter wavelengths;

- 2251+158 (3C 454.3): In this quasar $(z=0.859$, highly SLM), two small outbursts appeared since 1994. Its modulation index increases with decreasing wavelength.

\section{Summary}

We have presented the data from a long-term flux monitoring project carried out with the Effelsberg radio telescope, on a complete sample of 13 flat spectrum sources from the S5 Survey and 27 other sources. Our observational findings can be summarized as follows:

- Nearly all but the two steep spectrum sources $0835+580$ and $0951+699$ (excluding the six primary calibrator sources), are variable at most of the observing wavelengths in this 5.5 years monitoring period;

- Among the 32 variable sources, 15 of the 19 IDV sources $(79 \%)$ show a relatively strong degree of variability (of above $\sim 7 \%$ at $6 \mathrm{~cm}$ ) and pronounced outbursts or significant flux variations. The rest 4 of the $19,0153+744,0917+624,1652+398$ and $1928+738$, do not show strong long-term variability. On the other hand, most of the non-IDV sources ( 6 of the 9 sources, i.e. $67 \%$ ) show a relatively weak variability (of below $\sim 7 \%$ at $6 \mathrm{~cm}$ ), but 2 sources $0212+735$ and $1226+023$ show different variability behavior at different wavelengths. This implies that IDV sources vary more strongly. Therefore we suggest that, one might identify an IDV source by its long-term variability characteristics (degree of variability and outbursts);

- As seen from Fig. 28, there is no dependence of the degree of variability on either source galactic latitude, or redshift, or superluminal motions; but both the degree of variability and the rate of occurrence of variability are higher when source spectra are flatter. It appears that there is a weak correlation between the degree of variability and the 6 to $2.8 \mathrm{~cm}$ spectral index;

- There is a trend showing that the degree of variability decreases with increasing wavelength, as demonstrated in Fig. 29, which is contrary to the expectations of ISS models, but consistent with shock models;

- The variability in BL Lac objects is stronger than that in quasars, as demonstrated in Fig. 30. Statistically, the mean variability at each wavelength in BL Lacs is about 2 times larger than that in QSOs. This is consistent with the unified scheme of the AGNs.

From this large although not complete sample, we conclude that, the long-term variability behavior does not follow the expectations of the ISS theory: the wavelength dependence and independence of the degree of variability on galactic latitude. Although it is generally accepted that the long term variations discussed here are intrinsic to the source, this result is a useful confirmation.

Acknowledgements. We thank I. Pauliny-Toth for critically reading the manuscript, the referee Dr. M.F. Aller for her valuable comments, R. Nan and Y. Han for helpful discussions, and A.P. Lobanov, E. Ros, J. Klare for helping with the observations. B. Peng thanks the NSF of China for supporting this research. This research has made use of the NASA's Astrophysics Data System Abstract Service and of the NASA/IPAC Extragalactic Database (NED) which is operated by the Jet Propulsion Laboratory, Caltech, under contract with the National Aeronautics and Space Administration.

\section{References}

Aller H.D., Aller M.F., Latimer G.E., Hodge P.E., 1985, ApJS 59,513

Baars J.W.M., Genzel R., Pauliny-Toth I.I.K., Witzel A., 1977, A\&A 61,99

Bevington P.R., 1969, Data Reduction and Error Analysis for the Physical Sciences. Mcgraw-Hill Book Company

Biretta J.A., Moore R.L., Cohen M.H., 1986, ApJ 308, 93

Blandford R.D., Königl A., 1979, ApJ 232, 34

Bloom S.D., Marscher A.P., Moore E.M., Gear W., et al., 1999, ApJS 122, 1

Bondi M., Padrielli L., Fanti R., Ficarra A., Gregorini L., Mantovani F., 1996, A\&AS 120, 89

Bregman J.N., Glassgold A.E., Huggins P.J., Neugebauer G., Soifer B.T., Matthews K., Elias H., et al., 1990, ApJ 352, 574

Britzen S., Witzel A., Krichbaum T.P., Qian S.J., Campbell R.M., 1999, A\&A 341, 418

Burbidge G.R., Jones T.W., O'Dell S.L., 1974, ApJ 193, 43

Chu H.S., Baath L.B., Rantakyrö F.T., Zhang F.J., Nicholson G., 1996, A\&A 307, 15

Cohen M.H., Zensus J.A., Biretta J.A., Comoretto G., Kaufmann P., Abraham Z., 1987, ApJ 315, L89

Cotton W.D., Counselman III C.C., Geller R.B., et al., 1979, ApJ 229, L115 
Dennison B., Broderick J.J., Ledden J.E., O’Dell S.L., Condon J.J., 1981, AJ 86, 1604

Eckart A., Witzel A., Biermann P., Pearson T.J., Readhead A.C.S., Johnston K.J., 1985, ApJ 296, L23

Fanti C., Fanti R., Ficarra A., Mantovani F., Padrielli L., Weiler K.W., 1981, A\&AS 45, 61

Gabuzda D.C., Cawthorne T.V., Roberts D.H., Wardle J.F.C., 1989, ApJ 347, 701

Gabuzda D.C., Cawthorne T.V., 1996, MNRAS 283, 759

Ghisellini G., Padovani P., Celotti A., Maraschi L., 1993, ApJ 407, 65

Gopal-Krishna, Singal A.K., Krishnamohan S., 1984, A\&A 140, L19

Heeschen D.S., Krichbaum T.P., Schalinski C.J., Witzel A., 1987, AJ 94, 1493

Hunstead R.W., 1972, Astrophys. Lett. 12, 193

Hough D.H., Zensus J.A., Vermeulen R.C., Readhead A.C.S., Porcas R.W., Rius A., 1993, in Subarcsecond Radio Astronomy, Davis R.J. and Booth R.S. (eds.). Cambridge Uni. Press, p. 195

Jones T.W., Burbidge G.R., 1973, ApJ 186, 791

Lang K.R., 1974, Astrophysical Formulae. Springer-Verlag

Kellermann K.I., Pauliny-Toth I.I.K., 1968, ARA\&A 6, 417

Kellermann K.I., 1974, in Galactic and Extra-Galactic Radio Astronomy, Verschuur G.L. \& Kellermann K.I. (eds.). Springer-Verlag New York Inc., p. 320

Kellermann K.I., Pauliny-Toth I.I.K., 1969, ApJ 155, L71

Kraus A., 1997, Ph.D. Thesis, University of Bonn

Kraus A., Quirrenbach A., Lobanov A., et al., 1999a, A\&A 344,807

Kraus A., Witzel A., Krichbaum T.P., Lobanov A., Peng B., Ros E., 1999b, A\&A 352, L107

Krichbaum T.P., Britzen S., Standke K.J., Witzel, A., Schalinski C.J., Zensus J.A., 1995, PNAS 92, 11377

Kühr H., Pauliny-Toth I.I.K., Witzel A., Schmidt J., 1981, AJ 86,854

Kus A.J., Wilkinson P.N., Pearson T.J., Readhead A.C.S., 1990, in Parsec-Scale Radio Jets, Zensus J.A. and Pearson T.J. (eds.). Cambridge Uni. Press, p. 161

Marscher A.P., Gear W.K., 1985, ApJ 298, 114

Mutel R.L., 1990, in Parsec-Scale Radio Jets, Zensus J.A. \& Pearson T.J. (eds.). Cambridge Univ. Press, p. 98

Otterbein K., Krichbaum T.P., Kraus A., et al., 1998, A\&A $334,489-497$
Padrielli L., Aller M.F., Aller H.D., et al., 1987, A\&AS 67, 63 Pauliny-Toth I.I.K., Porcas R.W., Zensus J.A., et al., 1987, Nat 328, 778

Peng B., Kraus A., Krichbaum T.P., et al., 2000, A\&A 353, 937

Qian S.J., 1996, Acta Astrophys. Sin. 16, 143

Quirrenbach A., Witzel A., Krichbaum T.P., Hummel C.A., Alberdi A., Schalinski C.J., 1989, Nat 337, 442

Quirrenbach A., Witzel A., Krichbaum T.P., et al., 1992, A\&A 258,279

Quirrenbach A., Kraus A., Witzel A., et al., 2000, A\&AS 141, 221

Rees M.J., 1966a, Nat 211, 468

Rees M., 1966b, MNRAS, 135, 345

Rickett B.J., 1986, ApJ 307, 564

Rickett, B.J., 1990, ARA\&A 28, 561

Romney J.D., Alef W., Pauliny-Toth I.I.K., Preuss E., Kellermann K.I., 1984, Fanti R., Kellermann K. and Setti G. (eds.), IAU Symp. 110, 137

Salonen E., Terasranta H., Urpo S., et al., 1987, A\&AS 70, 409

Schalinski C.J., Witzel A., Krichbaum T.P., Hummel C.A., Quirrenbach A., Jonstone K.J., 1992, in Variability in Blazars, Valtaoja E. and Valtonen M. (eds.). Cambridge Univ. Press, p. 221

Shaffer D.B., Marscher A.P., Marcaide J., Romney J.D., 1987, ApJ 314, L1

Spangler S.R., Cotton W.D., 1981, AJ 86, 730

Standke K.J., Quirrenbach A., Krichbaum T.P., et al., 1996, A\&A 306, 27

Stickel M., Meisenheimer K., Kühr H., 1994, A\&AS 105, 211

Teräsranta H., Tornikoski M., Valtaoja E., et al., 1992, A\&AS 94, 121

Wagner S.J., Witzel A., 1995, ARA\&A 33, 163

Wagner S.J., Witzel A., Heidt J., et al., 1996, AJ 111, 2187

Witzel A., Heeschen D.S., Schalinski C.J., Krichbaum T.P., 1986, Mitt. Astron. Ges. 65, 239

Witzel A., Schalinski C.J., Johnston K.J., et al., 1988, A\&A 206,245

Valtaoja E., Terasranta, Urpo S., Nesterov N.S., Lainela M., Valtonen M., 1992, A\&A 254, 71

Woltjer L., 1966, ApJ 146, 597

Zensus J.A., 1997, ARA\&A 35, 607 